\title{
DESIGN OF ANTI-PARALLELOGRAM LOOP ASSEMBLIES
}

\section{ŞEBNEM GÜR ${ }^{1}, K^{\prime} O R A Y$ KORKMAZ ${ }^{1}$ and GÖKHAN KIPER ${ }^{2}$}

\author{
${ }^{1} \dot{I}_{z m i r}$ Institute of Technology, Faculty of Architecture, Department of Architecture \\ Gülbahçe Campus, 35430, Urla, İzmir \\ sebnemgur@iyte.edu.tr \\ koraykorkmaz@iyte.edu.tr \\ ${ }^{2}$ Izmir Institute of Technology, Faculty of Engineering, Department of Mechanical Engineering \\ Gülbahçe Campus, 35430, Urla, İzmir \\ gokhankiper@iyte.edu.tr
}

Editor's Note: Manuscript submitted 27 March 2019; revision received 31 August; accepted 12 September 2019. This paper is open for written discussion, which should be submitted to the IASS Secretariat no later than March 2020.

DOI: https://doi.org/10.20898/j.iass.2019.201.006

\section{ABSTRACT}

Scissor mechanisms are frequently used for deployable structures and many studies have been conducted on the subject. Most of the studies consider scissor units as modules in the design process. An alternative approach is to utilize loops as the modules for design. In this paper, the design alternatives of single degree-of-freedom planar linkages comprising anti-parallelogram loops using the loop assembly method is presented. First, scissor mechanisms are reviewed. Next, the types of four-bar loops and the resulting linkages in the literature are introduced and those which are yet to be explored, anti-parallelogram being one of them, are identified. Then the loop assembly method and the examples in the literature are reviewed. As a method to form as many alternatives as possible, symmetry operations are proposed. Suitable frieze symmetry groups utilized for obtaining the assemblies are explained and the anti-parallelogram loop patterns are derived. Next, the single degree-of-freedom linkages are obtained from the loop assemblies. Finally, a selection of the resulting linkages with novel properties are presented. This study shows that loop assemblies are efficient in systematic type synthesis of scissor linkages, some types of which could not be foreseen by using units as modules.

Keywords: deployable structures, scissor mechanisms, anti-parallelogram loop, loop assembly method

\section{INTRODUCTION}

"Deployable Structure" refers to mechanisms that can transform between a compact stowed and a deployed functional configuration [1]. They are also known as erectable, expandable, extendible, developable or unfurlable structures [2]. Applications of deployable structures can be seen in many fields such as aerospace industry as space antennas and masts [3], in architecture as retractable roofs [4-6] and kinetic building skins [7], and in engineering as bridges [8-11] and lifts. They attract a substantial attention, due to their capacity of motion, the simplicity of their elements and stowing efficiency. This entails many research to be conducted on the subject. Scissor mechanisms are one of the foremost choices to construct deployable structures.

Scissor mechanisms used in large-scale architectural designs were first introduced by Spanish architect
Emilio Perez Piñero in the early 1960s [12] and many researchers followed his lead from then on. Despite the subject has been extensively studied, it is still possible to find new approaches to the design.

Scissor units are made up of two ternary links, called the scissor-like elements (SLEs), connected with an intermediate revolute joint. If these units are connected at their terminal joints, a planar deployable linkage is achieved. The position of the intermediate joint together with the shape of the bars lead to three main scissor units commonly noted in the literature: translational, polar and angulated units. Common approach of designing scissor linkages is focusing on the scissor units and treating them as modules to create linkages, as Escrig [13], Glassner [14], Gantes et al. [15] and You and Pellegrino [16] did.

As an alternative, a 4-sided loop formed by two adjacent scissor units can be considered as a module. 
Unlike the unit based method, loop assembly method starts with the final form of the linkage to form the links specific to the configuration. Hoberman was the first to use the method. As a result of his work, Hoberman came up with angulated scissor units, which was a significant contribution to the field. Common loop types examined in the literature are shown in Figure 1, with green and blue colored links making up each scissor pair and grey colored loops. a)

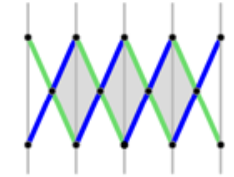

Rhombus loops

b)

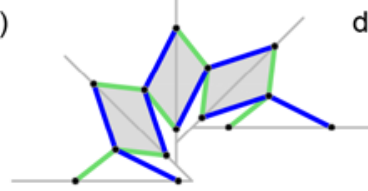

Parallelogram loops c)

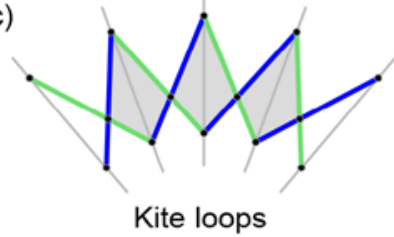

d)

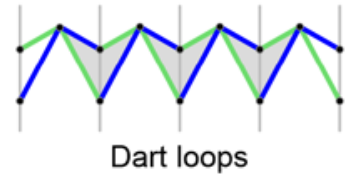

Figure 1: Common loop types in the literature

This paper is organized as follows: In the first section loop assembly method to synthesize deployable linkages is explained. In the second section, as a method for loop multiplication, Frieze symmetry group operations are introduced. Next, methods to define links for single degree-of-freedom linkage formation from the loop arrays is explained. In the last section selected linkages due to their novel properties are presented in detail.

\section{LOOP ASSEMBLY METHOD}

It was Hoberman who devised a method to form the links using loops $[17,18]$. While designing the linkage, he aligns identical rhombus loops on a curve such as a circle to derive the links (Fig. 2).
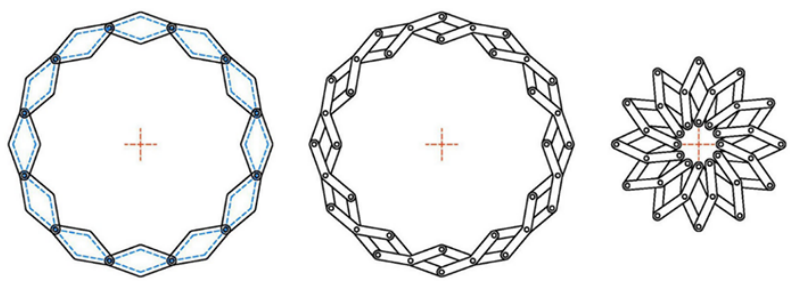

Figure 2: Assembly of rhombi loops on a circle [Hoberman et al., 2013]

This way, he is able to form angulated units with constant subtended angles. The unit lines intersect at the center of the circle. The same method is also proved to be effective when used on curves with variable curvature such as an ellipse. Expanding his research, Hoberman experimented with similar rhombus loops placed on a polygon which also yielded deployable scaling mechanisms. In this design, every edge of the polygon is made up of one rhombus loop scaled to fit. After Hoberman, Liao and Li [19] and Kiper and Söylemez [20] also used rhombus loops to design scaling linkages for planar graphs and polygon. Mao et al. issued assembly of parallelogram loops in order to obtain double chain linkages [21].

In 2014 Bai et al. utilized similar parallelogram, kite and general tetragon loops in addition to rhombus loops to construct polygonal scaling mechanisms [22]. They experimented with connections between various nodes of identical loops and provided a list of assembly options for the four loop types that can be used for scaling mechanisms. Another research using this method is conducted by Yar et al. [23] who used kite and dart loops aligned on a line with parallel unit lines to form transformable single DoF planar linkages. The linkages designed are able to transform from a linear form to concave and convex curves. Latest research on loop assemblies are published by the authors, present anti-parallelogram loop (Fig. 3) assemblies in various configurations [24-26]. These assemblies are special cases of one of the findings of this study and can be found under Section 5 - Linkage 4.
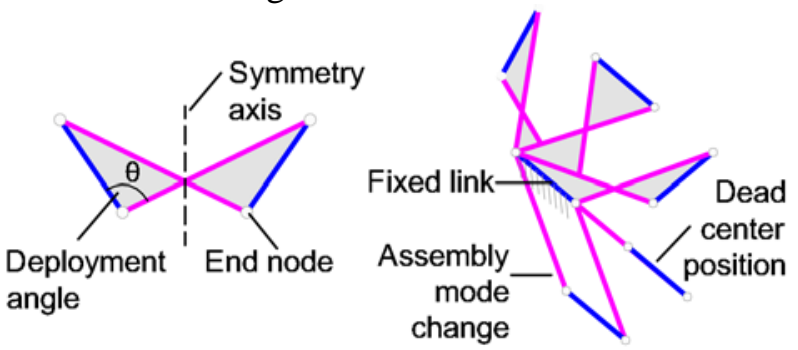

Figure 3: Linkage with anti-parallelogram loop and its motion

\section{FRIEZE PATTERNS}

Studies of loop assemblies in the literature mainly aim to form scalable linkages. Hoberman uses rhombus loops which are symmetric, therefore, has only two alternatives of connection. Later, Bai et al. [22] focus on the connection alternatives of several types of loops, however there is no systematic approach for deriving the loop patterns. Yar et al. [23] experiment with arrays of similar and different kite loops but do not investigate possible loop patterns. In order to generate possible loop assemblies, symmetry operations are used in this study. Although symmetries in a 2D plane may 
consist of multiplications on more than one direction, such as wallpaper groups, the aim of forming a curve-like linkage restricts the options with those that are multiplied in one direction only. This restriction led the research to Frieze patterns $[27,28]$. There are 7 array types of Frieze patterns (Fig. 4). Frieze patterns are combinations of horizontal reflection, vertical reflection, 180 degree rotation and glide reflection with repeated translations along a line.

Although Frieze patterns consist of seven types, in this study five of the variations are examined and two

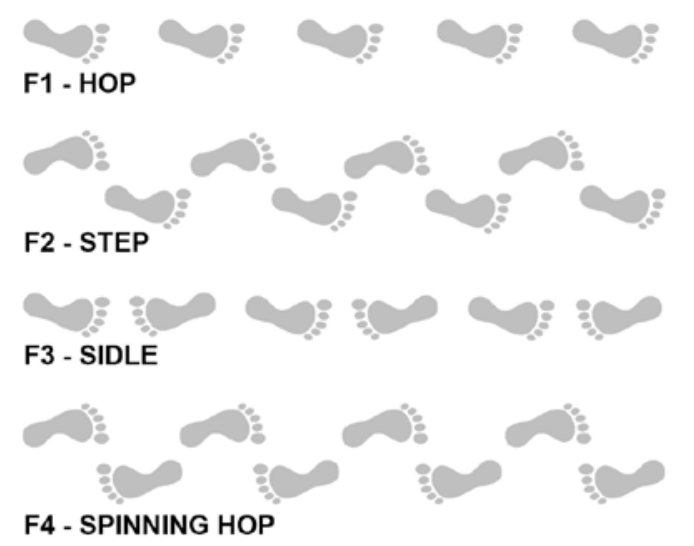

are eliminated. The reason is, 'F6 - Jump' and 'F7 Spinning Jump' patterns are made of two rows of items connected to each other (Fig. 4), which would correspond to two parallel connected series of linkages. Such a formation is not aimed in this study.

An anti-parallelogram has four corners which also represents joints of a loop of a linkage. When the loops are multiplied, they are attached at these joints. Depending on the direction of translation it is possible to formulate alternative arrays for each Frieze group operation. The cross-rectangle is a special condition of an anti-parallelogram that has

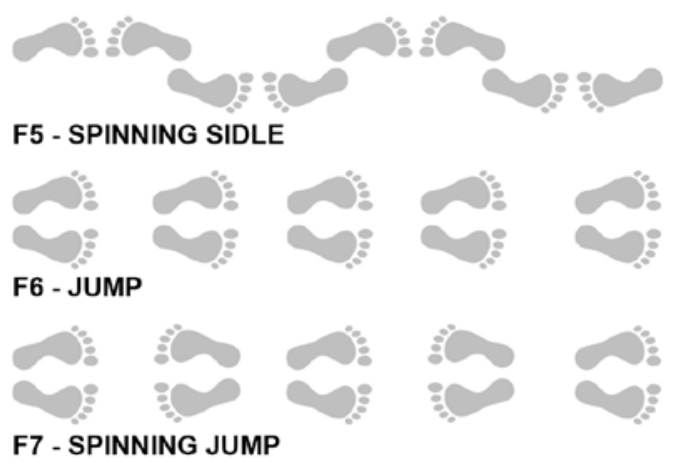

Figure 4: Frieze Groups [Redrawn from xploreandxpress.blogspot.com, by KNM]

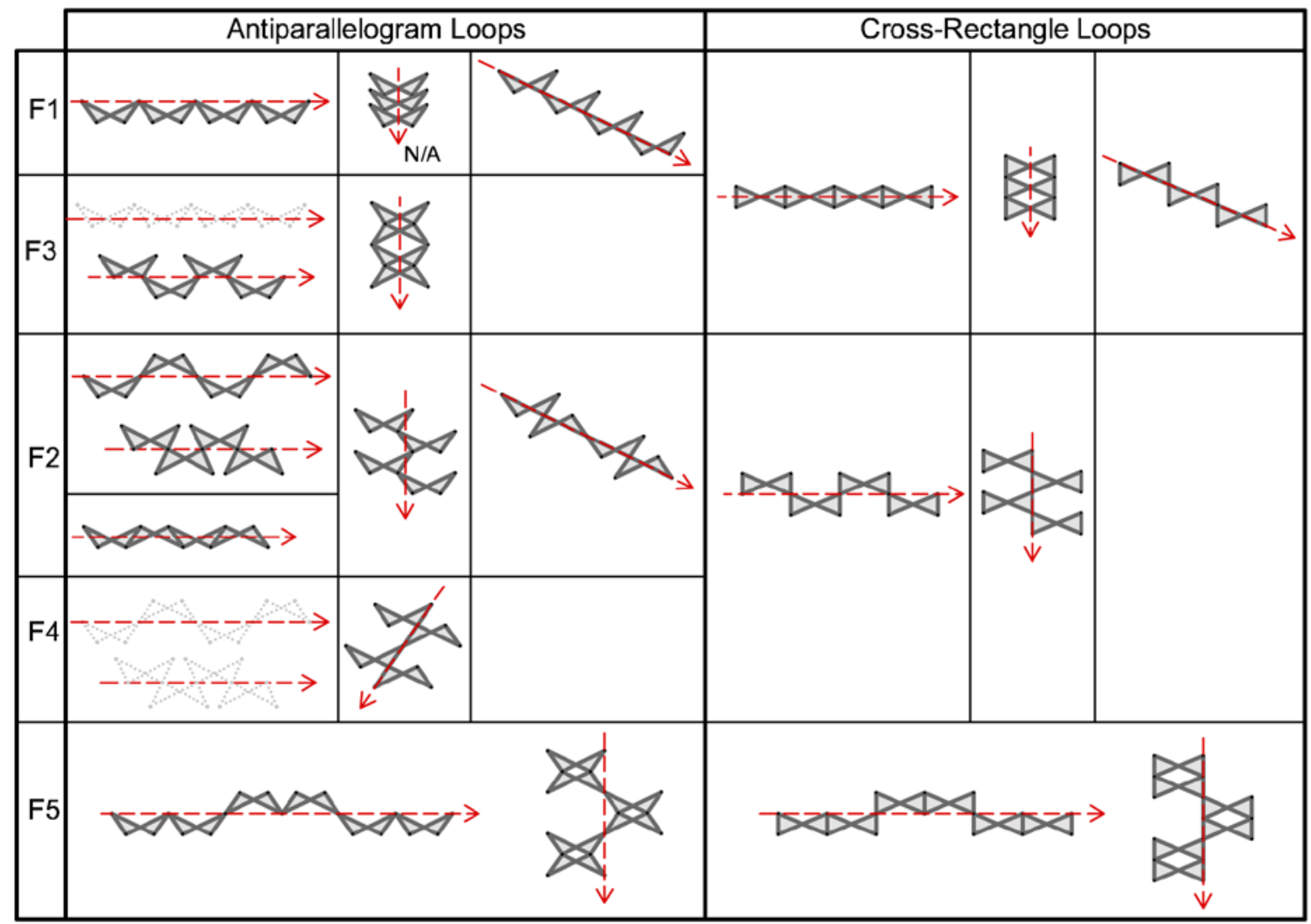

Figure 5: Frieze Group symmetries of anti-parallelogram and cross-rectangle 


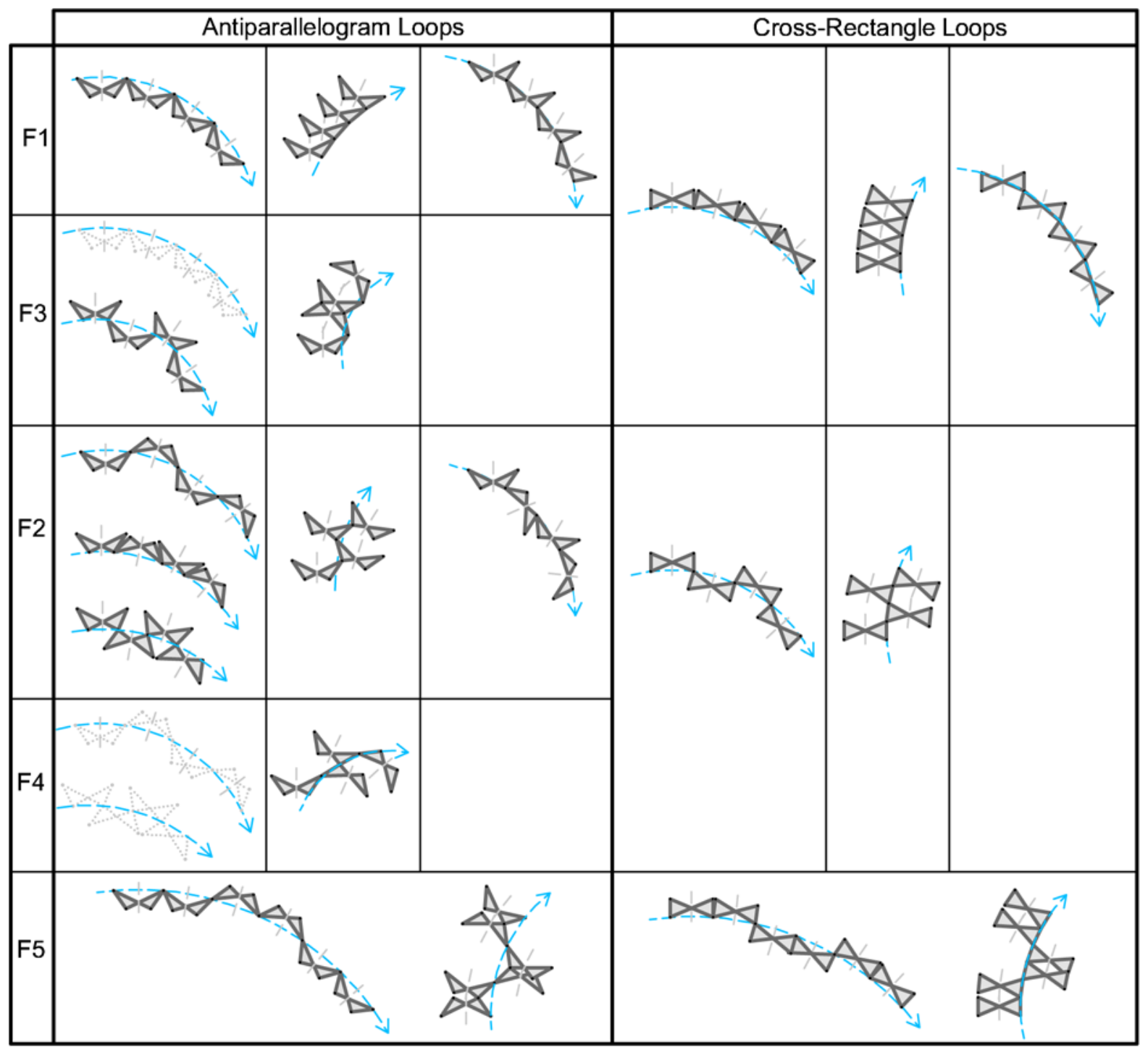

Figure 6: Frieze Group symmetries of anti-parallelogram and cross-rectangle on a curve

parallel short sides. When patterns of crossrectangles are obtained with Frieze groups, special cases which result in having straight links, are possible. The vertical mirror symmetry of an antiparallelogram and the vertical and horizontal mirror symmetries of cross-rectangle reduce the number of array alternatives significantly. Fig. 5 shows the Frieze array alternatives on a line for antiparallelogram and cross-rectangle loops. Array alternatives on a curve are shown in Fig. 6. Arrays resulting in the same as a prior one are shown with dotted lines

\section{FORMING SINGLE DOF LINKAGES}

In order to achieve single degree-of-freedom linkages, two links from each adjacent loop should be combined to form two common links. There are two options, either connect a long side with the short side of the neighboring loop or connect long sides and short sides within themselves (Fig. 7). However, the combination choice is not the only variation.

A rotational array may lead to special cases for which due to some specific rotation angle, sides of the loops may be collinear thus resulting in straight bar links (Fig. 8). The difference in link formation yields various motion capabilities. All configuration variations were modelled in Solidworks ${ }^{\circledR}$ to observe their motion. Few of the alternatives resulted in interesting results due to their motion capacity.

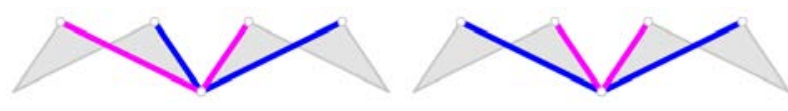

Figure 7: Alternative link formations between two loops 


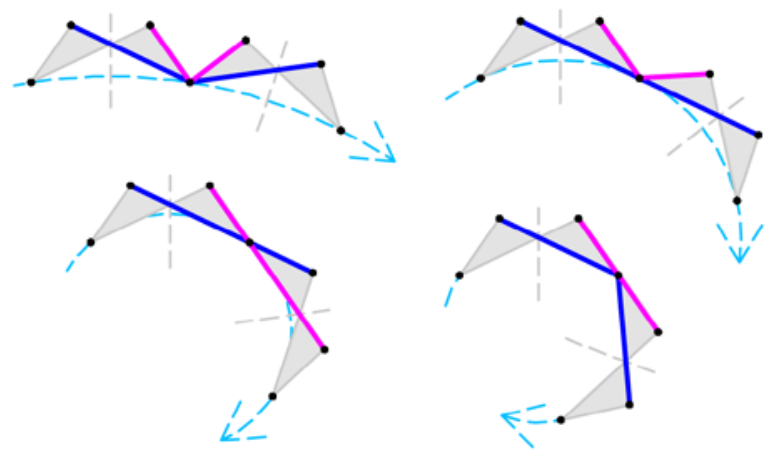

Figure 8: Frieze Group symmetries of anti-parallelogram on a curve

\section{SELECTED LINKAGES}

\subsection{Linkage 1}

The array in Fig. 9 is formed using the 'F2 - Step' group. It consists of cross-rectangles which are repeated along a line in one-over, one-under formation. The links formed are identical angulated ternary links. Due to the symmetric placement of the loops within the array, every other loop deploys similarly.

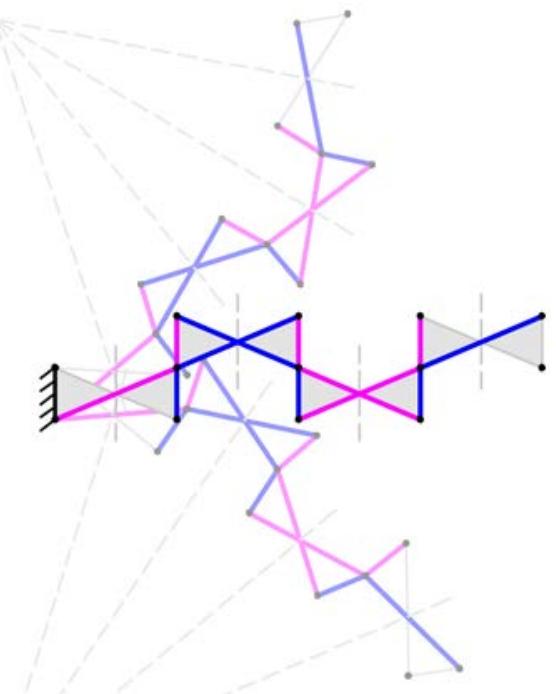

Figure 9: Array typology and motion of Linkage-1

A unit line is described as the line passing through the end points on the same side of a scissor pair and the motion capability of the linkage is defined by the changes of the angle between two unit lines of the link pair. A significant characteristics of a linkage with anti-parallelogram loops is that unit lines do not provide any meaningful geometrical property of the linkage. Instead, the symmetry axis of the loop yields the true nature of the linkage, able to present the curvature and deployment characteristics. Making use of the symmetry axis, it is seen that this linkage can be transformed from convex to concave form. During deployment the curvature of the linkage changes and only at the start position the curvature is zero.

\subsection{Linkage 2}

The array in Fig. 10 is formed using the 'F1 - Hop' group. It consists of cross-rectangles which are repeated along a line in a diagonal formation. The links formed are angulated. However, in the diagonal configuration, one link of a loop is common to that of one before and one after it, turning it into a quaternary link.

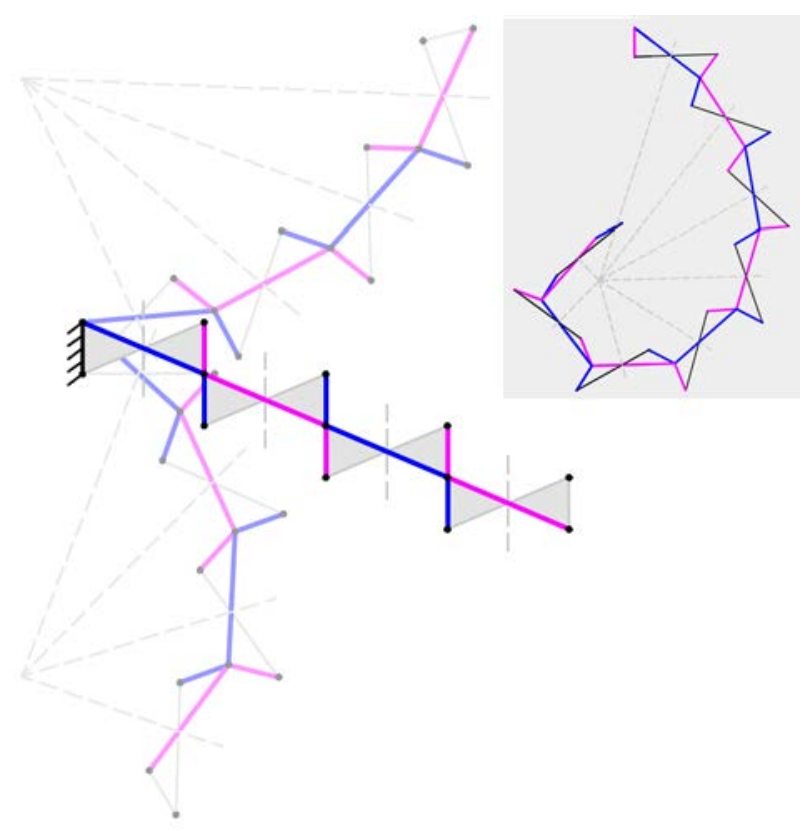

Figure 10: Array typology and motion of Linkage-2

When deployed, the linkage assumes a spiral form in either direction, which can be better observed when the number of loops is increased as seen in the grey area in Fig. 10. The intersection of the symmetry axes is the center of the spiral. This point goes to infinity when the linkage is at the initial configuration and the loops are parallel.

\subsection{Linkage 3}

The array in Fig. 11 is formed using the 'F1 - Hop' group on a circular arc. Using this array type there are many linkage alternatives to be formed. The rotation angle of the loops in the array leads to straight bars at specific points, as seen in Fig. 8. As the forms of the links differ, so does the motion capabilities of the linkages. The linkage in Fig. 12 is 
made up of identical straight bars. They are connected with an eccentrically placed intermediate joint, just like a polar unit. However, unlike a polar scissor linkage, when two units come together in this novel linkage, the short side is attached to the long side of the next unit.

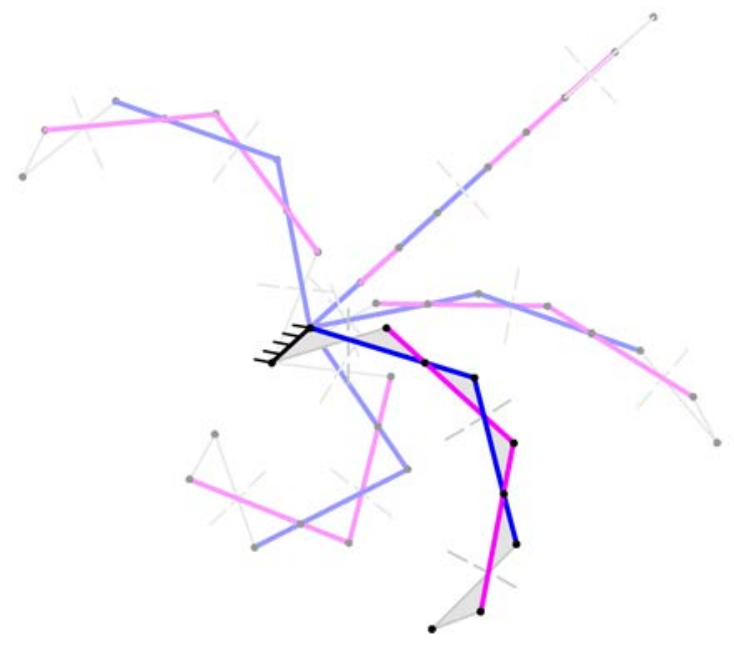

Figure 11: Array typology and motion of Linkage-2

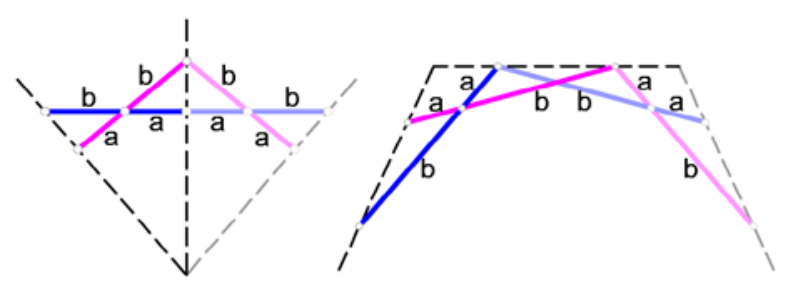

Figure 12: Polar scissor pair compared to antiparallelogram loop link pair

When the linkage in Fig. 11 is deployed, it is seen that it can transform from convex to concave form. This kind of movement hasn't been observed in linkages with straight bars before.

While transforming from concave to convex form, at one point all the links become collinear, assuming the dead center position, which might result in assembly mode change.

\subsection{Linkage 4}

The array in Fig. 13 is formed using the 'F2 - Step' group with rotation. It consists of cross-rectangles which are repeated along a circular arc in one-over, one-under formation. Both links are angulated ternary links with equal arms but the lengths are different for each link.

During motion, the symmetry axes preserve their relative positions. The curve defined for the array is divided equally by the loops. Therefore, preservation of the symmetry axes also means that each joint (which is also the point where loops are joined) positioned on the curve also move on an axis drawn from the intersection of symmetry axes. The subtended angles of the symmetry axes remain constant while the radius of the curve changes. In the light of these findings, a ring assembly is formed (Fig. 14). The details of this linkage is presented in [25].

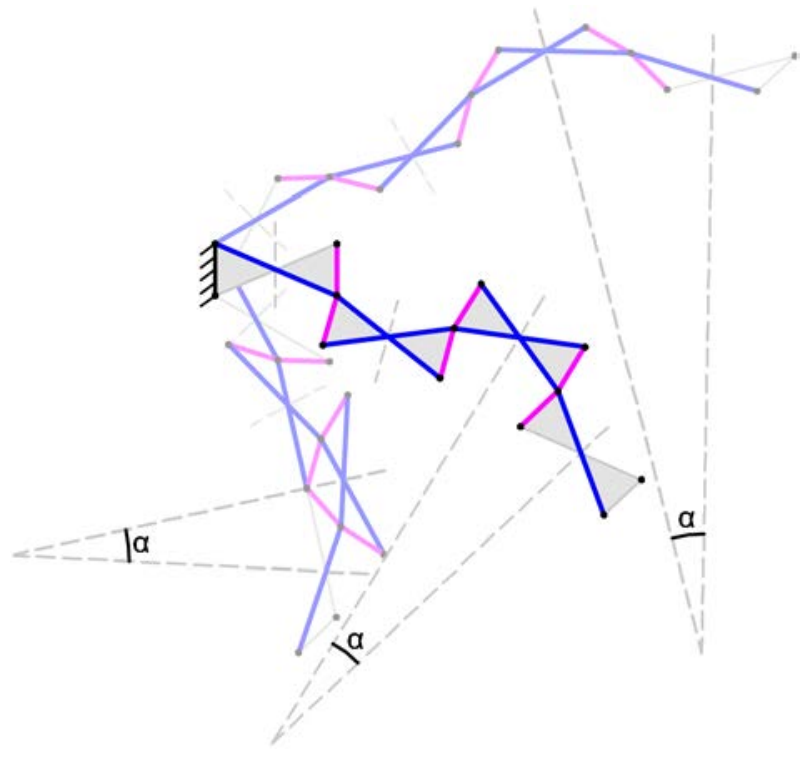

Figure 13: Array typology and motion of Linkage-4

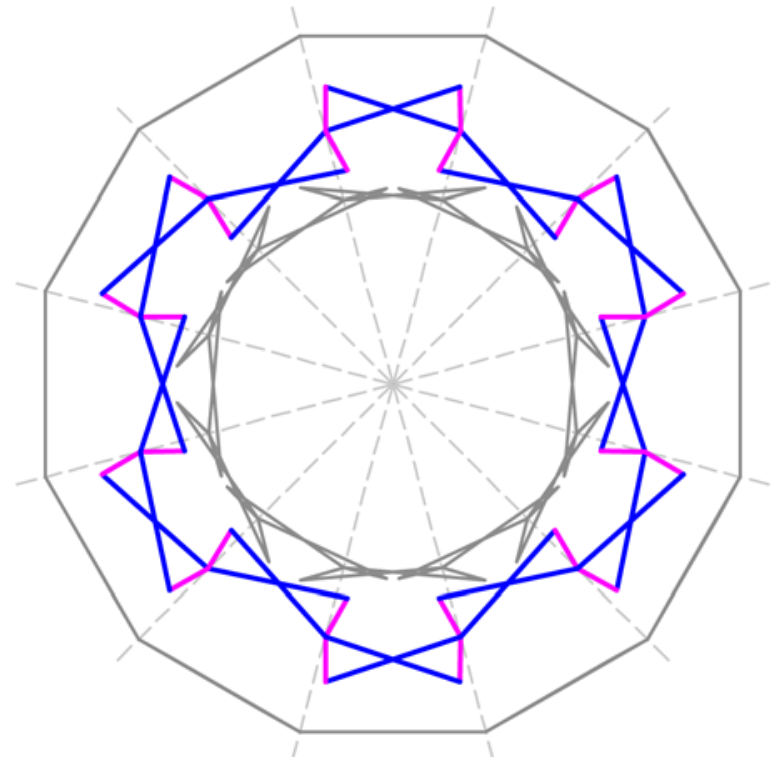

Figure 14: Array typology and motion of Linkage-4

At the fully-expanded state, the linkage forms a polygon, short and long links of each loop aligning on top of each other. At this state, the edge length of the polygon is the sum of one arm of each short and 
long links. The links formed in this linkage are one short and one long ternary angulated links with same kink angle and equal arms, connected by an intermediate joint at the kink point. They are similar to the angulated scissors but as in Linkage 3, they connect different than the other assemblies with angulated scissor units in the literature. The links can be classified as Type II GAE defined by You \& Pellegrino.

For the linkages examined so far, equal size loops are used and hence the curve is divided into equal segments. In his rhombus assemblies, Hoberman uses not only identical loops, but similar loops at different scales, such as in his patent [17]. Using the same principle, another assembly has been made with different scale loops (Fig. 15) [25]. Simulation of the linkage in Solidworks ${ }^{\circledR}$ shows that such a mechanism is also deployable. Depending on the neighboring angles, link pairs with various kink angles and arm lengths are produced. They are represented with color variations in Fig. 15. The links formed in the linkage are again classified as Type II GAE defined by You \& Pellegrino.

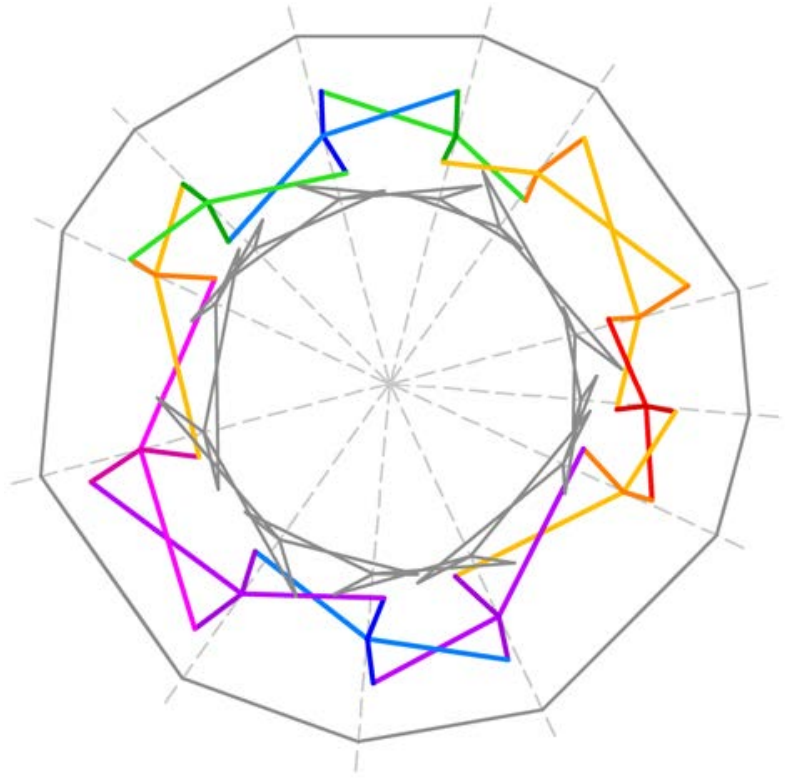

Figure 15: Ring structure with varying sizes of the same loop

The findings on the deployability of linkages with similar loops as in the ring assembly case suggest that all linkages examined in the previous cases could also be formed with similar loops. However, the analyses of such linkages are kept out of scope of this study.

\section{CONCLUSION}

Aim of this study was to create novel deployable and transformable planar structural mechanisms using loop assembly method and anti-parallelogram loops.

To be able to produce arrays in a methodical way, symmetry operations were examined. Frieze Group operations are found to be suitable for the aim of the research. After this step, single DoF linkages were formed. Within many linkages produced, four of them were selected due to their novel aspects.

Linkages 1, 2 and 3 all perform a transformation from convex to straight and then to concave form, a novel motion type from the previous linkages produced with loop assemblies, while they all have different types of links. Especially, Linkage 2 is the first linkage in the literature, with straight bars just like polar scissor units, observed to move in this fashion.

As the starting point of the research was loop assembly method utilized by Hoberman, another variation in his study was also examined. The ring assembly of Linkage 4 is composed again, this time with different scales of the same loop. It is seen that; such an assembly was also deployable.

Future studies may involve exploring more of the linkages defined in this study in detail. Seeking potential uses in architectural and engineering applications may also be considered as next step. In a kinematic point of view, exploration of possible linkages in three dimensional space could be another field to be studied.

\section{ACKNOWLEDGMENTS}

This project has received funding from the European Union's Horizon 2020 research and innovation programme under the Marie Skłodowska-Curie grant agreement No 689983.

\section{REFERENCES}

[1] H. M. C. H., "Deployable Structures," MSc. Thesis, Department of Architecture, Massachusetts Institute of Technology, Cambridge, MA, 1987.

[2] F. V. Jensen, "Concepts for Retractable Roof Structures," Ph.D. Dissertation, Department of Engineering, University of Cambridge, Cambridge, UK, 2004. 
[3] G. Kiper and E. Soylemez, "Deployable Space Structures," in International Conference on Recent Advances in Space Technologies: Proceedings of the 4th Rast 2009, Istanbul, Turkey, June 11-13 2019, pp. 131-138.

(DOI: 10.1109/Rast.2009.5158183)

[4] J. G. Cai, Y. H. Zhou, Y. F. Zhu, J. Feng, Y. $\mathrm{X}$. $\mathrm{Xu}$, and J. Zhang, "Geometry and Mechanical Behaviour of Radially Retractable Roof Structures During the Movement Process," (in English), International Journal of Steel Structures, vol. 16, no. 3, pp. 755-764, Sep 2016. (DOI: 10.1007/s13296-014-0173-7)

[5] G. Huntington Craig, "1.7 Movable and Retractable Roofs," American Society of Civil Engineers (ASCE), 2014.

[6] D. Mao and Y. Z. Luo, "Analysis and design of a type of retractable roof structure," (in English), Advances in Structural Engineering, vol. 11, no. 4, pp. 343-354, Aug 2008.

(DOI: 10.1260/136943308785836817)

[7] M. Pesenti, G. Masera, F. Fiorito, and M. Sauchelli, "Kinetic Solar Skin: A Responsive Folding Technique," (in English), International Conference on Solar Heating and Cooling for Buildings and Industry, Shc 2014, vol. 70, pp. 661-672, 2015. (DOI: 10.1016/j.egypro.2015.02.174)

[8] I. Ario, M. Nakazawa, Y. Tanaka, I. Tanikura, and S. Ono, "Development of a Prototype Deployable Bridge Based on Origami Skill," (in English), Automation in Construction, vol. 32, pp. 104-111, Jul 2013. DOI: 10.1016/j.autcon.2013.01.012

[9] E. Bouleau and G. Guscetti, "Scissor Mechanisms for Transformable Structures with Curved Shape," presented at the Advances in Architectural Geometry 2016, 2016. (DOI: $10.3218 / 3778-4)$

[10] G. Lederman, Z. You, and B. Glisic, "A Novel Deployable Tied Arch Bridge," (in English), Engineering Structures, vol. 70, pp. 1-10, Jul 1 2014. (DOI: 10.1016/j.engstruct.2014.03.015)

[11] Ş. Gür, M. Yar, and K. Korkmaz, "Çok fonksiyonlu form değiştirebilir köprü tasarımı için iki serbestlikli strüktürel mekanizma önerisi," Gazi Üniversitesi Mühendislik-Mimarlık Fakültesi Dergisi, vol. 32, no. 4, pp. 1379-1392, 2017. (DOI: 10.17341/gazimmfd.369866)

[12] E. Pinero, "Project for a Mobile Theatre," Architectural Design, vol. 12, no. 1, pp. 154155, 1961.

[13] F. Escrig, "Expandable Space Structures," International Journal of Space Structures, vol. 1, no. 2, pp. 79-91, 1985. (DOI: 10.1177/026635118500100203)

[14] A. Glassner, "Frieze Groups," IEEE Computer Graphics and Applications, vol. 16, no. 3, pp. 78-83, 1996. (DOI: $10.1109 / 38.491188$ )

[15] C. J. Gantes, J. J. Connor, R. D. Logcher, and Y. Rosenfeld, "Structural-Analysis and Design of Deployable Structures," (in English), Computers \& Structures, vol. 32, no. 3-4, pp. 661-669, 1989. (DOI: 10.1016/0045-7949(89)90354-4)

[16] Z. You and S. Pellegrino, "Foldable Bar Structures," (in English), International Journal of Solids and Structures, vol. 34, no. 15, pp. 1825-1847, May 1997. (DOI: 10.1016/S0020-7683(96)00125-4)

[17] C. Hoberman, "Reversibly Expandable Doubly-Curved Truss Structure," US Patent 4,942,700, 1990.

[18] C. Hoberman, E. Demaine, and D. Rus, "Mechanical invention through computation: expanding structures 2D," MIT Class, vol. 6, p. S080.

[19] Q. Liao and D. Li, "Mechanisms for Scaling Planar Graphs," Chinese Journal of Mechanical Engineering, vol. 8, p. 026, 2005.

[20] G. Kiper and E. Söylemez, "Irregular Polygonal and Polyhedral Linkages Comprising Scissor and Angulated Elements," in Asian Conference on Mechanism and Machine Science: Proceedings of the 1st IFToMM ASIAN MMS 2010, Taipei, Taiwan, October 21-25, 2010, pp. 455-459, NY: Curran Associates, Inc., 2011. 
[21] D. Mao, Y. Luo, and Z. You, "Planar closed loop double chain linkages," Mechanism and Machine Theory, vol. 44, no. 4, pp. 850859, 2009. (DOI:

10.1016/j.mechmachtheory.2008.04.005)

[22] G. Bai, S. Wei, D. Li, Q. Liao, and X. Kong, "A Novel Synthesis Method of PolygonScaling Mechanisms," in International Design Engineering Technical Conferences and Computers and Information in Engineering Conference: Proceedings of ASME IDETC/CIE 2014 Buffalo, New York, USA, August 17-20, 2014: American Society of Mechanical Engineers. (DOI: 10.1115/DETC2014-34934)

[23] M. Yar, K. Korkmaz, G. Kiper, F. Maden, Y. Akgün, and E. Aktaş, "A Novel Planar Scissor Structure Transforming Between Concave And Convex Configurations," International Journal of Computational Methods and Experimental Measurements, vol. 5, no. 4, pp. 442-450, 2017. (DOI: 10.2495/CMEM-V5-N4-442-450)

[24] Ş. Gür, C. Karagöz, G. Kiper, and K. Korkmaz, "Synthesis of Scalable Planar Scissor Linkages with Anti-parallelogram Loops," in European Conference on Mechanism Science: Proceedings of 7 th
IFTOMM EUCOMES 2018, Aachen, Germany, September 4-6, 2018, pp. 417424: Springer. (DOI: 10.1007/978-3-31998020-1_49)

[25] Ş. Gür, K. Korkmaz, and G. Kiper, "Radially Expandable Ring-Like Structure with Antiparallelogram Loops," in International Symposium of Mechanism and Machine Science: Proceedings of IFToMM ISMMS 2017, Baku, Azerbaijan, September 11-14, 2017, pp. 150-155

[26] S. Gür, M. Yar, and K. Korkmaz, "A Novel Two Degrees-of-Freedom Structural Mechanism Proposal for Multi-Functional Transformable Bridge," Journal of the Faculty of Engineering and Architecture of Gazi University, vol. 32, no. 4, pp. 13791392, 2017. (DOI: 10.1007/978-3-31998020-1_49)

[27] G. Pólya, "XII. Über die Analogie der Kristallsymmetrie in der Ebene," Zeitschrift für Kristallographie - Crystalline Materials, vol. 60, no. 1-6, p. 278, 1924. (DOI: 10.1524/zkri.1924.60.1.278)

[28] J. H. Conway, H. Burgiel, and C. GoodmanStrauss, The symmetries of things. AK Peters/CRC Press, 2016.

(DOI: 10.1201/b21368) 